\title{
Latex particle agglutination for detecting and identifying Clostridium difficile
}

\author{
RA BOWMAN, SUZAN A ARROW, TV RILEY
}

From the Department of Microbiology, University of Western Australia, Queen Elizabeth II Medical Centre, Nedlands, Western Australia

SUMMARY A total of 329 selective enrichment broth cultures were tested for detection of Clostridium difficile by latex particle agglutination (LPA), gas-liquid chromatography, and bacterial culture. Of 53 broths positive by LPA, 36 were positive by gas-liquid chromatography, and 42 were positive by bacterial culture. The sensitivity and specificity of LPA relative to bacterial culture was $95.6 \%$ and $96.3 \%$, respectively, while the sensitivity and specificity of gas-liquid chromatography relative to bacterial culture was $84.6 \%$ and $100 \%$, respectively. The high predictive value of a negative test (99\%) should make LPA on broth cultures a good screening test for detecting $C$ difficile. Of several other Clostridium spp tested in pure culture, strains of $C$ sordellii and $C$ bifermentans also gave a positive result by LPA. These results, together with the low cost and simple facilities required, suggest that the LPA test will be a useful procedure for the presumptive identification of $C$ difficile in selective enrichment broths and for the identification of pure cultures.

Clostridium difficile has a well documented role in pseudomembranous colitis and diarrhoea associated with antibiotics. ${ }^{1}$ Evidence has also implicated this organism in colitis not induced as a result of antibiotics, ${ }^{2}$ exacerbations of chronic inflammatory bowel disease, ${ }^{3}$ and postoperative diarrhoea. ${ }^{4}$ Many workers rely on the detection of $C$ difficile cytotoxin in stool samples before attaching any importance to the isolation of $C$ difficile. ${ }^{5}$ In other investigations, however, the finding of faecal cytotoxin was not consistent. ${ }^{6}$ C difficile may persist in stool samples for some time after cytotoxin is no longer detectable, ${ }^{8}$ particularly after treatment. In addition, many small laboratories may not have the facilities to detect C difficile cytotoxin. In view of these facts we, and others, have suggested that isolating $C$ difficile in addition to showing the presence of faecal cytotoxin may be important in the diagnosis and management of disease associated with $C$ difficile. ${ }^{910}$

We recently reported the use of a selective broth (GCC broth) that improved our isolation rate for C difficile by $20 \%$ in patients in whose faeces cytotoxin was detected and $125 \%$ in patients in whose faeces cytotoxin was not detected. 9 A presumptive identification of the presence of $C$ difficile in GCC broth was based on showing a large isocaproic acid peak by gas-liquid chromatography. As gas-liquid chromatography is relatively expensive and unavailable to small laboratories an alternative pro-

Accepted for publication 31 October 1985 cedure was sought. In this report we describe the use of a commercially available latex particle agglutination (LPA) test for the detection and rapid identification of $C$ difficile.

\section{Material and methods}

BACTERIAL STRAINS

A total of 60 Clostridium spp were obtained from the culture collections of either the University Department of Microbiology or the State Health Laboratory Service. They comprised the following: one Cabsonum (ATCC 27555); one Cbifermentans (NCTC 506); one Cbutyricum (NCTC 7424); 42 $C$ difficile (all clinical isolates); one $C$ fallax (NCTC 8380); two Chistolyticum (NCTC 503 and NCTC 7123); one Cparaperfringens (ATCC 27639); two Cperfringens (NCTC 8237 and NCTC 8359); one C septicum (NCTC 547); one C sphenoides (NCTC 507); four $C$ sordellii (all clinical isolates); one $C$ sporogenes (NCTC 532); one C tertium (NCTC 541); and one $C$ tetanomorphum (NCTC 2909). Lyophilised cultures were reconstituted and grown for $\mathbf{4 8}$ hours on blood agar plates incubated anaerobically using the Gaspak system (BBL Microbiology Systems, Cockeysville).

\section{SAMPLES}

A total of 329 stool samples were obtained from two sources; patients who were either inpatients or outpatients at Sir Charles Gairdner Hospital (a 700 bed 
general hospital); and patients who were seen by their general practitioners in either rural centres throughout Western Australia, an area of one million square miles, or the metropolitan area of the capital city, Perth. Specimens from hospital patients were normally processed within two hours of collection. Other specimens were usually cultured within 24 hours of collection. Due to the isolation of some rural centres (up to 1500 miles away) some longer delays were unavoidable. $C$ difficile remains viable in stool samples for at least four days (unpublished observations).

\section{CULTURAL PROCEDURES AND CYTOTOXIN DETECTION}

The methods used for isolating $C$ difficile and showing the presence of $C$ difficile cytotoxin have been described previously. ${ }^{7}$ They included the use of a selective broth for $C$ difficile, containing gentamicin, cycloserine, and cefoxitin (GCC broth). ${ }^{9}$ Final identification of $C$ difficile was done according to the methods and criteria of Holdeman, Cato, and Moore. $^{11}$

LATEX PARTICLE AGGLUTINATION (LPA) TEST The Serobact $C$ difficile latex slide agglutination kit was supplied by Disposable Products, Adelaide, South Australia. Each kit contained latex particles that had been coated with an immunoglobulin $\mathbf{G}$ specific for $C$ difficile cell wall antigens and a suspension of $C$ difficile to be used as a positive control. The test was carried out on black cardboard tiles that were supplied with the kit. Fresh subcultures of Cdifficile on CCFA $^{12}$ were used for assessment. When testing colonies from solid media a smooth suspension of organism was made in one drop (about $0.02 \mathrm{ml}$ ) of saline and observed for autoagglutination. One drop of $C$ difficile latex reagent was added to this suspension, or to one drop of GCC broth culture. After mixing the slide was gently rocked and observed for agglutination for up to two minutes before discarding.

\section{Results}

The results of testing from solid media were as follows: all 42 strains of $C$ difficile reacted strongly with the $C$ difficile latex reagent. All reactions were clear cut and easy to interpret. Of the 13 other species of clostridia tested, all four strains of $C$ sordellii and the one $C$ bifermentans gave a positive result.

A total of $329 \mathrm{GCC}$ broths were tested for the presumptive identification of Cdifficile. Thirty six broths were positive by bacterial culture, gas-liquid chromatography, and LPA. In six broths LPA was positive and bacterial culture yielded $C$ difficile; gasliquid chromatography analysis, however, was nega-
Percentage sensitivity, specificity, and predictability of positive or negative results

\begin{tabular}{|c|c|c|c|}
\hline & $G L C-B C^{*}$ & $L P A-B C \dagger$ & $L P A-G L C_{+}^{+}$ \\
\hline $\begin{array}{l}\text { Sensitivity } \\
\text { Specificity }\end{array}$ & $\begin{array}{l}84 \cdot 6 \\
100\end{array}$ & $\begin{array}{l}95 \cdot 6 \\
96 \cdot 3\end{array}$ & $\begin{array}{l}100 \\
94 \cdot 5\end{array}$ \\
\hline $\begin{array}{l}\text { (positive) } \\
\text { Predictability }\end{array}$ & 100 & 80 & $67 \cdot 9$ \\
\hline (negative) & $97 \cdot 3$ & 99 & 100 \\
\hline
\end{tabular}

*Gas-liquid chromatography compared with bacterial culture.

†Latex particle agglutination compared with bacterial culture.

† Latex particle agglutination compared with gas-liquid chromatography.

tive. In a further two broths bacterial culture alone was the only positive variable, while in 11 broths LPA was the only positive variable.

The Table shows the sensitivity, specificity, and predictability of gas-liquid chromatography and LPA. If bacterial culture is taken as the reference method then the sensitivity of gas-liquid chromatography and LPA was $84.6 \%$ and $95.6 \%$, respectively. The predictability of a positive result with gas-liquid chromatography was $100 \%$, while for LPA it was $80 \%$. Similarly, the predictability of a negative result for gas-liquid chromatography and LPA was $97.3 \%$ and $99 \%$, respectively.

Sensitivity, specificity, and predictability of a positive and negative test for LPA compared with gasliquid chromatography was $100 \%, 94.5 \%, 67.9 \%$, and $100 \%$, respectively.

\section{Discussion}

In a previous report we showed that the isolation rate of $C$ difficile from patients with diarrhoeal disease could be considerably improved by using a selective broth. ${ }^{9}$ The screening of these broths, however, was done using gas-liquid chromatography, a technique not available to many laboratories. The advent of a commercially available kit for the detection of $C$ difficile by LPA should allow small laboratories to improve their isolation rate by using a selective broth.

Although the identification of $C$ difficile is relatively easy using prereduced anaerobically sterilised media, ${ }^{11}$ such procedures may also be unavailable in small laboratories. In addition, further delays of up to 72 hours after primary isolation may occur using this method of identification. The $C$ difficile latex agglutination test permits rapid identification from a primary selective medium such as CCFA.

The results of the bacterial culture of GCC broths showed greater agreement with those of the LPA than the results of the broths with gas-liquid chromatography. There were, however, still 11 broths that were positive by LPA but negative by bacterial culture. These discrepant results could be either true false 
positives, or they could reflect enhanced sensitivity of LPA. CCFA has been reported as having a sensitivity of $10^{2}$ organisms/g faeces, although direct stool culture on solid selective media often gives variable results. ${ }^{12}$ Alternatively, some $C$ difficile strains may have failed to reach a sufficiently high concentration in GCC broth. We noticed that Streptococcus faecalis, which is not suppressed by GCC broth, may inhibit the growth of $C$ difficile strains. Inhibition of the multiplication of $C$ difficile by various enteric bacteria, particularly $S$ faecalis, has been reported previously. ${ }^{13}$ The most likely reason for the false positive LPA result, however, is cross reactions with other clostridia. Five (four $C$ sordellii and one $C$ bifermentans) of the 18 different Clostridium spp tested gave a positive LPA result. Thus an antigen common to these species could be responsible for the apparent false positive results. Strong cross reactions of antigens of $C$ difficile, $C$ sordelli, and $C$ bifermentans have been observed previously. ${ }^{14}$ Attempts to isolate either $C$ sordellii or $C$ bifermentans from false positive LPA broths were unsuccessful; their presence in low numbers, however, could not be excluded. Several broths yielded a microaerophilic Streptococcus species on culture, which was subsequently shown to react with $C$ difficile latex reagent.

Several recent papers have described rapid techniques for the detection of faecal cytotoxin such as counterimmunoelectrophoresis ${ }^{15}$ and LPA $^{16}$ for diagnosing diarrhoea associated with $C$ difficile. Other investigators ${ }^{17}$ concluded that the high percentage of false positive and false negative results with counterimmunoelectrophoresis makes this technique unsuitable for screening stool specimens. Furthermore, they suggested that bacterial isolation, together with the cytotoxicity assay, was the most accurate method for the detection of $C$ difficile. The high predictive value of a negative result made the cytotoxin LPA a useful screening test. ${ }^{16}$ As the detection of faecal cytotoxin was not a consistent feature in disease associated with C difficile ${ }^{67}$ we find this conclusion difficult to justify. There have also been reports of simple methods for the detection of $C$ difficile enterotoxin such as enzyme linked immunosorbent assay. ${ }^{1819}$ In one of these ${ }^{18}$ the sensitivity for specimens with a positive faecal cytotoxin assay was only $59 \%$.

Hence our efforts ${ }^{9}$ and those of others ${ }^{10}$ have been directed towards improving techniques for the isolation of $C$ difficile. We used the familiar technique of selective broth culture for isolation of an enteric pathogen. The procedure has now been simplified considerably, however, by replacing the gas-liquid chromatography with an easy LPA test, enabling small laboratories to improve their isolation rate by using a selective broth. The high predictive value of a negative result $(99 \%)$ will allow these laboratories to confidently use LPA on GCC broths as a screening test. If required, positive broths may then be forwarded to a central laboratory for further testing. The isolation of $C$ difficile from or the detection of cyto- toxin in stool samples does not necessarily constitute a diagnosis of pseudomembranous colitis or diarrhoea associated with antibiotics. Once detected, however, the importance of any isolate, either cytotoxigenic or non-cytotoxigenic, can then be assessed taking into consideration the patient's clinical condition.

We thank V Wymer, V Bamford, and J Iveson of the Public Health and Enteric Disease Unit of the State Health Laboratory Service for their cooperation.

\section{References}

${ }^{1}$ Bartlett JG, Chang TW, Gurwith M, Gorbach SL, Onderdonk AB. Antibiotic-associated pseudomembranous colitis due to toxinproducing clostridia. $N$ Engl J Med 1978;298:531-4.

${ }^{2}$ Howard JM, Sullivan SN, Troster M. Spontaneous pseudomembranous colitis. Br Med J 1980;281:356.

${ }^{3}$ Bolton RP, Sherriff RJ, Read AE. Clostridium difficile associated diarrhoea: a role in inflammatory bowel diseases. Lancet 1980;i:383-4.

${ }^{4}$ Keighley MRB, Burdon DW, Alexander-Williams J, et al. Diarrhoea and pseudomembranous colitis after gastrointestinal operations. Lancet 1978;ii:1165-7.

${ }^{5}$ Laughon BE, Viscidi RP, Gdovin SL, Yolken RH, Bartlett JG. Enzyme immunoassays for detection of Clostridium difficile toxins $A$ and $B$ in fecal specimens. $J$ Infect Dis 1984;149:781-8.

${ }^{6}$ Mollby R, Nord CE, Aronsson B. Diagnosis of Clostridium difficile-associated enterocolitis in Sweden: laboratory and epidemiological aspects. Scand J Infect Dis 1980;22(suppl):30-6.

${ }^{7}$ Riley TV, Bowman RA, Carroll SM. Diarrhoea associated with Clostridium difficile in a hospital population. Med J Aust 1983;:166-9.

${ }^{8}$ Teasley DG, Olson MM, Gebhard RL, et al. Prospective randomised trial of metronidazole versus vancomycin for Clostridium difficile-associated diarrhorea and colitis. Lancet 1983;ii:1043-6.

${ }^{9}$ Carroll SM, Bowman RA, Riley TV. A selective broth for Clostridium difficile. Pathology 1983;15:165-7.

${ }^{10}$ Levett PN. Use of enrichment cultures for the isolation of Clostridium difficile from stools. Microbios Letts 1984;25:67-9.

${ }^{11}$ Holdeman LV, Cato EP, Moore WEC. Anaerobe laboratory manual. 4th ed. Blacksburg: Virginia Polytechnic Institute and State University, 1977.

${ }^{12}$ George WL, Sutter VL, Citron D, Finegold SM. Selective and differential medium for isolation of Clostridium difficile. J Clin Microbiol 1979;9:214-9.

${ }^{13}$ Malamou-Ladas H, Tabaqchali S. Inhibition of Clostridium difficile by faecal streptococci. J Med Microbiol 1980;15:569-74.

${ }^{14}$ Poxton IR, Byrne MD. Immunochemical analysis of the EDTAsoluble antigens of Clostridium difficile and related species. $J$ Gen Microbiol 1981;122:41-6.

${ }^{15}$ Ryan RW, Kwasnik I, Tilton RC. Improved immunologic detection of Clostridium difficile antigen by counterimmunoelectrophoresis. Diagn Microbiol Infect Dis 1983;1:59-63.

${ }^{16}$ Shahrabadi MS, Bryan LE, Gaffrey D, Coderre SE, Gordon R, Pai $\mathrm{CH}$. Latex agglutination test for detection of Clostridium difficile toxin in stool samples. J Clin Microbiol 1984;20:339-41.

${ }^{17}$ Jarvis W, Nunez-Montiel O, Thompson F, et al. Comparison of bacterial isolation, cytotoxicity assay, and counterimmunoelectrophoresis for the detection of Clostridium difficile and its toxin. $J$ Infect Dis 1983;147:778.

${ }^{18}$ Lyerly DM, Sullivan NM, Wilkins TD. Enzyme-linked immunosorbent assay for Clostridium difficile toxin A.J Clin Microbiol 1983;17:72-8.

${ }^{19}$ Laughon BE, Viscidi RP, Gdovin SL, Yolken RH, Bartlett JG. Enzyme immunoassays for detection of Clostridium difficile toxins A and B in faecal specimens. J Infect Dis 1984;149:781-8.

Requests for reprints to: Dr TV Riley, Department of Microbiology, University of Western Australia, Queen Elizabeth II Medical Centre, Nedlands, Western Australia 6009. 